\title{
Semileptonic Decays of $B\left(B_{s}\right)$ to Light Tensor Mesons
}

\author{
Reza Khosravi and S. Sadeghi \\ Department of Physics, Isfahan University of Technology, Isfahan 84156-83111, Iran \\ Correspondence should be addressed to Reza Khosravi; rezakhosravi@cc.iut.ac.ir
}

Received 23 June 2016; Revised 21 August 2016; Accepted 6 September 2016

Academic Editor: Juan José Sanz-Cillero

Copyright (C) 2016 R. Khosravi and S. Sadeghi. This is an open access article distributed under the Creative Commons Attribution License, which permits unrestricted use, distribution, and reproduction in any medium, provided the original work is properly cited. The publication of this article was funded by SCOAP ${ }^{3}$.

\begin{abstract}
The semileptonic $B_{s}(B) \rightarrow K_{2}^{*}\left(a_{2}, f_{2}\right) \ell v, \ell=\tau, \mu$, transitions are investigated in the frame work of the three-point QCD sum rules. Considering the quark condensate contributions, the relevant form factors of these transitions are estimated. The branching ratios of these channel modes are also calculated at different values of the continuum thresholds of the tensor mesons and compared with the obtained data for other approaches.
\end{abstract}

\section{Introduction}

Investigation of the $B$ meson decays into tensor mesons is useful in several aspects such as CP asymmetries, isospin symmetries, and the longitudinal and transverse polarization fractions. A large isospin violation has already been experimentally detected in $B \rightarrow \omega K_{2}^{*}$ (1430) mode [1]. Also, the decay mode $B \rightarrow \phi K_{2}^{*}(1430)$ is mainly dominated by the longitudinal polarization $[2,3]$, in contrast with $B \rightarrow \phi K^{*}$, where the transverse polarization is comparable with the longitudinal one [4]. Therefore, nonleptonic and semileptonic decays of $B$ meson can play an important role in the study of the particle physics.

In the flavor $S U$ (3) symmetry, the light $p$-wave tensor mesons with $J^{P}=2^{+}$containing isovector mesons $a_{2}$ (1320), isodoublet states $K_{2}^{*}(1430)$, and two isosinglet mesons $f_{2}(1270)$ and $f_{2}^{\prime}(1525)$ are building the ground state nonet which has been experimentally established $[5,6]$. The quark content $q \bar{q}$ for the isovector and isodoublet tensor resonances is obvious. The isoscalar tensor states, $f_{2}(1270)$ and $f_{2}^{\prime}(1525)$, have mixing wave functions where mixing angle should be small $[7,8]$. Therefore, $f_{2}(1270)$ is primarily a $(u \bar{u}+d \bar{d}) / \sqrt{2}$ state, while $f_{2}^{\prime}(1525)$ is dominantly $s \bar{s}$ [9].

As a nonperturbative method, the QCD sum rules is a well established technique in the hadron physics since it is based on the fundamental QCD Lagrangian [10]. The semileptonic decays of $B$ to the light mesons involving $\pi$,
$K\left(K^{*}, K_{0}^{*}\right)$, and $a_{1}$ have been studied via the three-point QCD sum rules (3PSR), for instance, $B \rightarrow \pi \ell v[11], B \rightarrow K \ell^{+} \ell^{-}$, $B \rightarrow K^{*} \ell^{+} \ell^{-}[12-14], B_{s} \rightarrow K_{0}^{*} \ell v[15], B_{s} \rightarrow\left(K_{0}^{*}, f_{0}\right) \ell^{+} \ell^{-}$ [16], and $B \rightarrow a_{1} \ell^{+} \ell^{-}$[17]. The determination of the form factor value $T_{1}(0)=0.35 \pm 0.05$ relevant for the $B \rightarrow K^{*} \gamma$ and $B \rightarrow K^{*} \ell^{+} \ell^{-}[14,18]$ decays allowed prediction of the ratio $\Gamma\left(B \rightarrow K^{*} \gamma\right) / \Gamma(b \rightarrow s \gamma)=0.17 \pm 0.05$, which agrees with the experimental measurements [19-21]. The obtained results of the decay $B \rightarrow \pi \ell \nu$ [11] and simulations on the lattice [22-24] are in a reasonable agreement.

In this work, we investigate $B\left(B_{s}\right) \rightarrow K_{2}^{*}\left(a_{2}, f_{2}\right) \ell v$ decays within the 3PSR method. For analysis of these decays, the form factors and their branching ratio values are calculated. So far, the form factors of the semileptonic decays $B\left(B_{s}\right) \rightarrow$ $K_{2}^{*}\left(a_{2}, f_{2}\right) \ell v$ have been studied via different approaches such as the LCSR [25], the perturbative QCD (PQCD) [5], the large energy effective theory (LEET) [26-28], and the ISGW II model [29]. A comparison of our results for the form factor values in $q^{2}=0$ and branching ratio data with predictions obtained from other approaches, especially the LCSR, is also made.

The plan of the present paper is as follows: the 3PSR approach for calculation of the relevant form factors of $B\left(B_{s}\right) \rightarrow K_{2}^{*}\left(a_{2}, f_{2}\right) \ell v$ decays is presented in Section 2. In the final section, the value of the form factors in $q^{2}=0$ and the branching ratio of the considered decays are reported. For a better analysis, the form factors and differential branching 


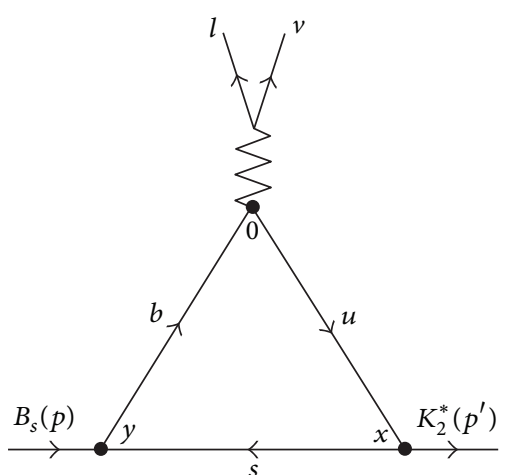

FIGURE 1: Schematic picture of the spectator mechanism for the $B_{s} \rightarrow K_{2}^{*} \ell v$ decay.

ratios related to these semileptonic decays are plotted with respect to the momentum transfer squared $q^{2}$.

\section{Theoretical Framework}

In order to study $B\left(B_{s}\right) \rightarrow K_{2}^{*}\left(a_{2}, f_{2}\right) \ell v$ decays, we focus on the exclusive decay $B_{s} \rightarrow K_{2}^{*}$ via the 3PSR. The $B_{s} \rightarrow$ $K_{2}^{*} \ell v$ decay governed by the tree level $b \rightarrow u$ transition (see Figure 1). In the framework of the 3PSR, the first step is appropriate definition of correlation function. In this work, the correlation function should be taken as

$$
\begin{aligned}
& \Pi_{\alpha \beta \mu}\left(p^{2}, p^{\prime 2}, q^{2}\right)=i \iint e^{i\left(p^{\prime} x-p y\right)}\langle 0| \\
& \mathscr{T}\left\{j_{\alpha \beta}^{K_{2}^{*}}(x) j_{\mu}(0) j^{B_{s}}(y)\right\}|0\rangle d^{4} x d^{4} y,
\end{aligned}
$$

where $p$ and $p^{\prime}$ are four-momentum of the initial and final mesons, respectively. $q^{2}$ is the squared momentum transfer and $\mathscr{T}$ is the time ordering operator. $j_{\mu}=\bar{u} \gamma_{\mu}\left(1-\gamma_{5}\right) b$ is the transition current. $j^{B_{s}}$ and $j_{\alpha \beta}^{K_{2}^{*}}$ are also the interpolating currents of $B_{s}$ and the tensor meson $K_{2}^{*}$, respectively. With considering all quantum numbers, their interpolating currents can be written as follows [33]:

$$
\begin{aligned}
& j^{B_{s}}(y)=\bar{b}(y) \gamma_{5} s(y), \\
& j_{\alpha \beta}^{K_{2}^{*}}(x) \\
& \quad=\frac{i}{2}\left[\bar{s}(x) \gamma_{\alpha} \stackrel{\leftrightarrow}{D}_{\beta}(x) u(x)+\bar{s}(x) \gamma_{\beta} \stackrel{\leftrightarrow}{D}_{\alpha}(x) u(x)\right],
\end{aligned}
$$

where $\stackrel{\leftrightarrow}{D}_{\mu}(x)$ is the four-derivative vector with respect to $x$ acting at the same time on the left and right. It is given as

$$
\begin{aligned}
& \stackrel{\leftrightarrow}{D}_{\mu}(x)=\frac{1}{2}\left[\vec{D}_{\mu}(x)-\overleftarrow{D}_{\mu}(x)\right], \\
& \vec{D}_{\mu}(x)=\vec{\partial}_{\mu}(x)-i \frac{g}{2} \lambda^{a} \mathbf{A}_{\mu}^{a}(x), \\
& \overleftarrow{D}_{\mu}(x)=\overleftarrow{\partial}_{\mu}(x)+i \frac{g}{2} \lambda^{a} \mathbf{A}_{\mu}^{a}(x),
\end{aligned}
$$

where $\lambda^{a}$ and $\mathbf{A}_{\mu}^{a}(x)$ are the Gell-Mann matrices and the external gluon fields, respectively. It should be noted that the second current in (2) interpolates a spin 2 particle for massless quarks. In the general case, to describe a spin 2 state one has to use a current such that the trace of $j_{\alpha \beta}^{K_{2}^{*}}$ vanishes.

The correlation function is a complex function of which the imaginary part comprises the computations of the phenomenology and real part comprises the computations of the theoretical part (QCD). By linking these two parts via the dispersion relation, the physical quantities are calculated. In the phenomenological part of the QCD sum rules approach, the correlation function in (1) is calculated by inserting two complete sets of intermediate states with the same quantum numbers as $B_{s}$ and $K_{2}^{*}$. After performing four integrals over $x$ and $y$, it will be

$$
\Pi_{\alpha \beta \mu}=-\frac{\left\langle 0\left|j_{\alpha \beta}^{K_{2}^{*}}\right| K_{2}^{*}\left(p^{\prime}\right)\right\rangle\left\langle K_{2}^{*}\left(p^{\prime}\right)\left|j_{\mu}\right| B_{s}(p)\right\rangle\left\langle B_{s}(p)\left|j^{B_{s}}\right| 0\right\rangle}{\left(p^{2}-m_{B_{s}}^{2}\right)\left(p^{\prime 2}-m_{K_{2}^{*}}^{2}\right)}+\text { higher states. }
$$

In (4), the vacuum to initial and final meson state matrix elements is defined as

$$
\begin{aligned}
\left\langle 0\left|j_{\alpha \beta}^{K_{2}^{*}}\right| K_{2}^{*}\left(p^{\prime}, \varepsilon\right)\right\rangle & =f_{K_{2}^{*}} m_{K_{2}^{*}}^{2} \varepsilon_{\alpha \beta}, \\
\left\langle 0\left|j^{B_{s}}\right| B_{s}(p)\right\rangle & =-i \frac{f_{B_{s}} m_{B_{s}}^{2}}{\left(m_{b}+m_{s}\right)},
\end{aligned}
$$

where $f_{K_{2}^{*}}$ and $f_{B_{s}}$ are the leptonic decay constants of $K_{2}^{*}$ and $B_{s}$ mesons, respectively. $\varepsilon_{\alpha \beta}$ is polarization tensor of $K_{2}^{*}$. The transition current gives a contribution to these matrix elements and it can be parametrized in terms of some form factors using the Lorentz invariance and parity conservation. The correspondence between a vector meson and a tensor meson allows us to get these parametrizations in a comparative way (for more information see [5]). The parametrization of $B \rightarrow T$ form factors is analogous to the $B \rightarrow V$ case except that $\varepsilon$ is replaced by $\varepsilon_{T}$, as follows:

$$
\begin{aligned}
c_{V}\left\langle K_{2}^{*}\left(p^{\prime}, \varepsilon\right)\left|\bar{u} \gamma_{\mu}\left(1-\gamma_{5}\right) b\right| B_{s}(p)\right\rangle \\
=-i \varepsilon_{T \mu}^{*}\left(m_{B_{s}}+m_{K_{2}^{*}}\right) A_{1}\left(q^{2}\right) \\
\quad+i\left(p+p^{\prime}\right)_{\mu}\left(\varepsilon_{T}^{*} \cdot q\right) \frac{A_{2}\left(q^{2}\right)}{m_{B_{s}}+m_{K_{2}^{*}}}
\end{aligned}
$$




$$
\begin{aligned}
& +i q_{\mu}\left(\varepsilon_{T}^{*} \cdot q\right) \frac{2 m_{K_{2}^{*}}}{q^{2}}\left(A_{3}\left(q^{2}\right)-A_{0}\left(q^{2}\right)\right) \\
& +\epsilon_{\mu \nu \rho \sigma} \varepsilon_{T}^{* v} p^{\rho} p^{\prime \sigma} \frac{2 V\left(q^{2}\right)}{m_{B_{s}}+m_{K_{2}^{*}}}
\end{aligned}
$$

with $A_{3}\left(q^{2}\right)$

$$
\begin{aligned}
& \quad=\frac{m_{B_{s}}+m_{K_{2}^{*}}}{2 m_{K_{2}^{*}}} A_{1}\left(q^{2}\right)-\frac{m_{B_{s}}-m_{K_{2}^{*}}}{2 m_{K_{2}^{*}}} A_{2}\left(q^{2}\right), \\
& A_{0}(0)=A_{3}(0),
\end{aligned}
$$

where $q=p-p^{\prime}, P=p+p^{\prime}$, and $\varepsilon_{T \mu}^{*}=\left(p_{\lambda} / m_{B_{s}}\right) \varepsilon_{\mu \lambda}$. The factor $c_{V}$ accounts for the flavor content of particles: $c_{V}=\sqrt{2}$ for $a_{2}, f_{2}$ and $c_{V}=1$ for $K_{2}^{*}$ [34]. Inserting (5) and (6) in (4) and performing summation over the polarization tensor as

$$
\varepsilon_{\mu \nu} \varepsilon_{\alpha \beta}=\frac{1}{2} T_{\mu \alpha} T_{\nu \beta}+\frac{1}{2} T_{\mu \beta} T_{\nu \alpha}-\frac{1}{3} T_{\mu \nu} T_{\alpha \beta}
$$

where $T_{\mu \nu}=-g_{\mu \nu}+p_{\mu}^{\prime} p_{\nu}^{\prime} / m_{K_{2}^{*}}^{2}$, the final representation of the physical side is obtained as

$$
\begin{aligned}
& \Pi_{\alpha \beta \mu}=\frac{f_{B_{s}} m_{B_{s}}}{\left(m_{b}+m_{s}\right)} \\
& \cdot \frac{f_{K_{2}^{*}} m_{K_{2}^{*}}^{2}}{\left(p^{2}-m_{B_{s}}^{2}\right)\left(p^{\prime 2}-m_{K_{2}^{*}}^{2}\right)}\left\{V^{\prime}\left(q^{2}\right) i \epsilon_{\beta \mu \rho \sigma} p_{\alpha} p^{\rho} p^{\prime \sigma}\right. \\
& +A_{0}^{\prime}\left(q^{2}\right) p_{\alpha} p_{\beta} p_{\mu}^{\prime}+A_{1}^{\prime}\left(q^{2}\right) g_{\beta \mu} p_{\alpha} \\
& \left.+A_{2}^{\prime}\left(q^{2}\right) p_{\alpha} p_{\beta} p_{\mu}\right\}+ \text { higher states. }
\end{aligned}
$$

For simplicity in calculations, the following redefinitions have been used in (9):

$$
\begin{aligned}
& V^{\prime}\left(q^{2}\right)=\frac{V\left(q^{2}\right)}{m_{B_{s}}+m_{K_{2}^{*}}}, \\
& A_{0}^{\prime}\left(q^{2}\right)=-\frac{m_{K_{2}^{*}}\left(A_{3}\left(q^{2}\right)-A_{0}\left(q^{2}\right)\right)}{q^{2}}, \\
& A_{1}^{\prime}\left(q^{2}\right)=-\frac{\left(m_{B_{s}}+m_{K_{2}^{*}}\right)}{2} A_{1}\left(q^{2}\right), \\
& A_{2}^{\prime}\left(q^{2}\right)=\frac{A_{2}\left(q^{2}\right)}{2\left(m_{B_{s}}+m_{K_{2}^{*}}\right)} .
\end{aligned}
$$

Now, the QCD part of the correlation function is calculated by expanding it in terms of the OPE at large negative value of $q^{2}$ as follows:

$$
\begin{aligned}
\Pi_{\alpha \beta \mu}= & C_{\alpha \beta \mu}^{(0)} I+C_{\alpha \beta \mu}^{(3)}\langle 0|\bar{\Psi} \Psi| 0\rangle \\
& +C_{\alpha \beta \mu}^{(4)}\left\langle 0\left|G_{\rho \nu}^{a} G_{a}^{\rho v}\right| 0\right\rangle \\
& +C_{\alpha \beta \mu}^{(5)}\left\langle 0\left|\bar{\Psi} \sigma_{\rho \nu} T^{a} G_{a}^{\rho v} \Psi\right| 0\right\rangle+\cdots,
\end{aligned}
$$

where $C_{\alpha \beta \mu}^{(i)}$ are the Wilson coefficients, $I$ is the unit operator, $\bar{\Psi}$ is the local fermion field operator, and $G_{\rho v}^{a}$ is the gluon strength tensor. In (11), the first term is contribution of the perturbative and the other terms are contribution of the nonperturbative part.

To compute the portion of the perturbative part (Figure 1), using the Feynman rules for the bare loop, we obtain

$$
\begin{aligned}
& C_{\alpha \beta \mu}^{(0)}=-\frac{i}{4} \iint e^{i\left(p^{\prime} x-p y\right)}\left\{\operatorname { T r } \left[S_{s}(x-y) \gamma_{\alpha} \stackrel{\leftrightarrow}{D}_{\beta}(x)\right.\right. \\
& \left.\cdot S_{u}(-x) \gamma_{\mu}\left(1-\gamma_{5}\right) S_{b}(y) \gamma_{5}\right]+\operatorname{Tr}[\alpha \\
& \longleftrightarrow \beta]\} d^{4} x d^{4} y
\end{aligned}
$$

taking the partial derivative with respect to $x$ of the quark free propagators and performing the Fourier transformation and using the Cutkosky rules, that is, $1 /\left(p^{2}-m^{2}\right) \rightarrow-2 i \pi \delta\left(p^{2}-\right.$ $\left.m^{2}\right)$, imaginary part of $C_{\alpha \beta \mu}^{(0)}$ is calculated as

$$
\begin{aligned}
& \operatorname{Im}\left[C_{\alpha \beta \mu}^{(0)}\right]=\frac{1}{8 \pi} \int \delta\left(k^{2}-m_{s}^{2}\right) \delta\left((p+k)^{2}-m_{b}^{2}\right) \\
& \cdot \delta\left(\left(p^{\prime}+k\right)^{2}-m_{u}^{2}\right)\left(2 k+p^{\prime}\right)_{\beta} \operatorname{Tr}\left[\left(k+m_{s}\right)\right. \\
& \left.\cdot \gamma_{\alpha}\left(\not p^{\prime}+k+m_{u}\right) \gamma_{\mu}\left(1-\gamma_{5}\right)\left(\not p+k+m_{b}\right) \gamma_{5}\right]+\{\alpha \\
& \longleftrightarrow \beta\} d^{4} k,
\end{aligned}
$$

where $k$ is four-momentum of the spectator quark $s$. To solve the integral in (13), we will have to deal with the integrals such as $I_{0}, I_{\alpha}, I_{\alpha \beta}$, and $I_{\alpha \beta \mu}$ with respect to $k$. For example, $I_{\alpha \beta \mu}$ can be as

$$
\begin{aligned}
& I_{\alpha \beta \mu}\left(s, s^{\prime}, q^{2}\right)=\int\left[k_{\alpha} k_{\beta} k_{\mu}\right] \delta\left(k^{2}-m_{s}^{2}\right) \\
& \cdot \delta\left((p+k)^{2}-m_{b}^{2}\right) \delta\left(\left(p^{\prime}+k\right)^{2}-m_{u}^{2}\right) d^{4} k,
\end{aligned}
$$

where $s=p^{2}$ and $s^{\prime}=p^{\prime 2} . I_{0}, I_{\alpha}, I_{\alpha \beta}$, and $I_{\alpha \beta \mu}$ can be taken as an appropriate tensor structure as follows:

$$
\begin{aligned}
I_{0} & =\frac{1}{4 \sqrt{\lambda\left(s, s^{\prime}, q^{2}\right)}}, \\
I_{\alpha} & =B_{1}\left[p_{\alpha}\right]+B_{2}\left[p_{\alpha}^{\prime}\right],
\end{aligned}
$$




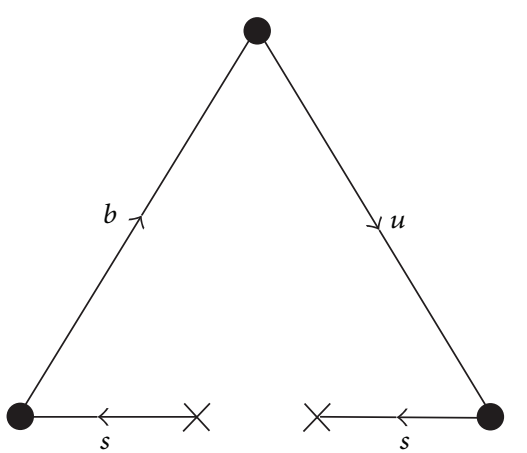

(a)

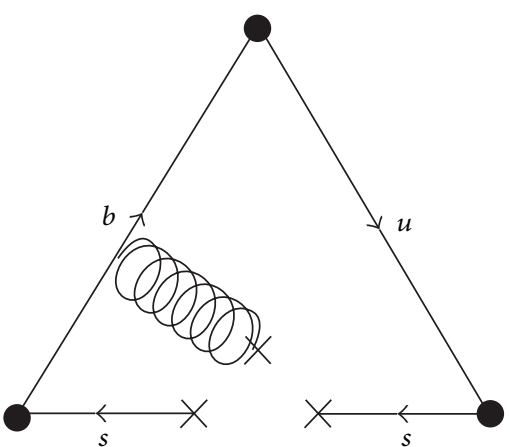

(b)

FIGURE 2: The diagrams of the effective contributions of the condensate terms.

$$
\begin{aligned}
I_{\alpha \beta}= & D_{1}\left[g_{\alpha \beta}\right]+D_{2}\left[p_{\alpha} p_{\beta}\right]+D_{3}\left[p_{\alpha} p_{\beta}^{\prime}+p_{\alpha}^{\prime} p_{\beta}\right] \\
& +D_{4}\left[p_{\alpha}^{\prime} p_{\beta}^{\prime}\right], \\
I_{\alpha \beta \mu}= & E_{1}\left[g_{\alpha \beta} p_{\mu}+g_{\alpha \mu} p_{\beta}+g_{\beta \mu} p_{\alpha}\right] \\
& +E_{2}\left[g_{\alpha \beta} p_{\mu}^{\prime}+g_{\alpha \mu} p_{\beta}^{\prime}+g_{\beta \mu} p_{\alpha}^{\prime}\right] \\
& +E_{3}\left[p_{\alpha} p_{\beta} p_{\mu}\right] \\
& +E_{4}\left[p_{\alpha}^{\prime} p_{\beta} p_{\mu}+p_{\alpha} p_{\beta}^{\prime} p_{\mu}+p_{\alpha} p_{\beta} p_{\mu}^{\prime}\right] \\
& +E_{5}\left[p_{\alpha}^{\prime} p_{\beta}^{\prime} p_{\mu}+p_{\alpha}^{\prime} p_{\beta} p_{\mu}^{\prime}+p_{\alpha} p_{\beta}^{\prime} p_{\mu}^{\prime}\right] \\
& +E_{6}\left[p_{\alpha}^{\prime} p_{\beta}^{\prime} p_{\mu}^{\prime}\right] .
\end{aligned}
$$

The quantities $\lambda\left(s, s^{\prime}, q^{2}\right), B_{l}(l=1,2), D_{j}(j=1, \ldots, 4)$, and $E_{r}(r=1, \ldots, 6)$ are indicated in Appendix. Using the relations in (15), $\operatorname{Im}\left[C_{\alpha \beta \mu}^{(0)}\right]$ can be calculated for each structure corresponding to (9) as follows:

$$
\begin{aligned}
\operatorname{Im}\left[C_{\alpha \beta \mu}^{(0)}\right]= & \rho_{V}\left(i \epsilon_{\beta \mu \rho \sigma} p_{\alpha} p^{\rho} p^{\prime \sigma}\right)+\rho_{0}\left(p_{\alpha} p_{\beta} p_{\mu}^{\prime}\right) \\
& +\rho_{1}\left(g_{\beta \mu} p_{\alpha}\right)+\rho_{2}\left(p_{\alpha} p_{\beta} p_{\mu}\right),
\end{aligned}
$$

where the spectral densities $\rho_{i}(i=V, 0,1,2)$ are found as

$$
\begin{aligned}
& \rho_{V}\left(s, s^{\prime}, q^{2}\right)=24 B_{1} \sqrt{\lambda}\left[B_{1}\left(m_{s}-m_{b}\right)\right. \\
& \left.\quad+B_{2}\left(m_{s}-m_{u}\right)+m_{s} I_{0}\right], \\
& \rho_{0}\left(s, s^{\prime}, q^{2}\right)=12\left[D_{2}\left(m_{s}-m_{b}\right)+D_{3}\left(m_{s}-m_{u}\right)\right. \\
& \left.\quad+2 B_{1} m_{s}-2 E_{4}\left(m_{b}-m_{s}\right)\right], \\
& \rho_{1}\left(s, s^{\prime}, q^{2}\right)=3 B_{1}\left[2 m_{s}^{2}\left(m_{b}+m_{u}-m_{s}\right)\right. \\
& \left.\quad-m_{s}\left(2 m_{b} m_{u}+u\right)+\Delta\left(m_{s}-m_{u}\right)+\Delta^{\prime}\left(m_{s}-m_{b}\right)\right] \\
& \quad+6 D_{1}\left(m_{s}-m_{u}\right)-24 E_{1}\left(m_{b}-m_{s}\right), \\
& \rho_{2}\left(s, s^{\prime}, q^{2}\right)=24\left[D_{2} m_{s}+E_{3}\left(m_{s}-m_{b}\right)\right] .
\end{aligned}
$$

Using the dispersion relation, the perturbative part contribution of the correlation function can be calculated as follows:

$$
C_{i}^{(0)}=\iint \frac{\rho_{i}\left(s, s^{\prime}, q^{2}\right)}{\left(s-p^{2}\right)\left(s^{\prime}-p^{\prime 2}\right)} d s^{\prime} d s .
$$

For calculation of the nonperturbative contributions (condensate terms), we consider the condensate terms of dimensions 3, 4, and 5 related to the contributions of the quark-quark, gluon-gluon, and quark-gluon condensate, respectively. They are more important than the other terms in the OPE. In the 3PSR, when the light quark is a spectator, the gluon-gluon condensate contributions can be easily ignored [35]. On the other hand, the quark condensate contributions of the light quark, which is a nonspectator, are zero after applying the double Borel transformation with respect to both variables $p^{2}$ and $p^{\prime 2}$, because only one variable appears in the denominator. Therefore, only two important diagrams of dimensions 3, 4, and 5 remain from the nonperturbative part contributions. The diagrams of these contributions corresponding to $C_{\alpha \beta \mu}^{(3)}$ and $C_{\alpha \beta \mu}^{(5)}$ are depicted in Figure 2. After some calculations, the nonperturbative part of the correlation function is obtained as follows:

$$
\begin{aligned}
C_{V}^{(3)}+C_{V}^{(5)}= & -\frac{2 \kappa}{\left(p^{2}-m_{b}^{2}\right)^{2}\left(p^{\prime 2}-m_{u}^{2}\right)}, \\
C_{0}^{(3)}+C_{0}^{(5)}= & -\frac{4 \kappa}{\left(p^{2}-m_{b}^{2}\right)^{2}\left(p^{\prime 2}-m_{u}^{2}\right)}, \\
C_{1}^{(3)}+C_{1}^{(5)}= & \frac{\kappa}{\left(p^{2}-m_{b}^{2}\right)\left(p^{\prime 2}-m_{u}^{2}\right)} \\
& +\frac{\kappa\left[\left(m_{b}+m_{u}\right)^{2}-q^{2}\right]}{\left(p^{2}-m_{b}^{2}\right)^{2}\left(p^{\prime 2}-m_{u}^{2}\right)}, \\
C_{2}^{(3)}+C_{2}^{(5)}= & -\frac{4 \kappa}{\left(p^{2}-m_{b}^{2}\right)^{2}\left(p^{\prime 2}-m_{u}^{2}\right)},
\end{aligned}
$$

where $\kappa=\left(\left(m_{s}^{2}-m_{0}^{2} / 2\right) / 16\right)\langle 0|s \bar{s}| 0\rangle, m_{0}^{2}=(0.8 \pm 0.2) \mathrm{GeV}^{2}$ [35], and $\langle 0|\bar{s} s| 0\rangle=(0.8 \pm 0.2)\langle 0|\bar{u} u| 0\rangle,\langle 0|\bar{u} u| 0\rangle=$ $\langle 0|\bar{d} d| 0\rangle=-(0.240 \pm 0.010 \mathrm{GeV})^{3}$; that is, we choose the 
TABLE 1: The values of the meson masses [30] and decay constants $[31,32] \mathrm{in} \mathrm{GeV}$.

\begin{tabular}{lccccc}
\hline Meson & $B_{s}$ & $B$ & $K_{2}^{*}$ & $a_{2}$ & $f_{2}$ \\
\hline Mass & 5.366 & 5.279 & 1.425 & 1.318 & 1.275 \\
Decay constant & $0.222 \pm 0.012$ & $0.186 \pm 0.014$ & $0.118 \pm 0.005$ & $0.107 \pm 0.006$ & $0.102 \pm 0.006$ \\
\hline
\end{tabular}

value of the condensates at a fixed renormalization scale of about $1 \mathrm{GeV}[36,37]$.

The next step is to apply the Borel transformations with respect to $p^{2}\left(p^{2} \rightarrow M_{1}^{2}\right)$ and $p^{\prime 2}\left(p^{\prime 2} \rightarrow M_{2}^{2}\right)$ on the phenomenological as well as the perturbative and nonperturbative parts of the correlation functions and equate these two representations of the correlations. The following sum rules for the form factors are derived:

$$
\begin{aligned}
& V^{\prime}\left(q^{2}\right)=\frac{\left(m_{b}+m_{s}\right) e^{m_{B_{s}}^{2} / M_{1}^{2}} e^{m_{K_{2}^{*}}^{2} / M_{2}^{2}}}{f_{B_{s}} m_{B_{s}} f_{K_{2}^{*}} m_{K_{2}^{*}}^{2}}\left\{\frac{-1}{(2 \pi)^{2}}\right. \\
& \cdot \int_{m_{s}^{2}}^{s_{0}^{\prime}} \int_{s_{L}}^{s_{0}} \rho_{V}\left(s, s^{\prime}, q^{2}\right) e^{-s / M_{1}^{2}} e^{-s^{\prime} / M_{2}^{2}} \\
& \left.+\widetilde{B}\left[C_{V}^{(3)}+C_{V}^{(5)}\right] d s^{\prime} d s\right\}, \\
& A_{n}^{\prime}\left(q^{2}\right)=\frac{\left(m_{b}+m_{s}\right) e^{m_{B_{s}}^{2} / M_{1}^{2}} e^{m_{K_{2}^{*}}^{2} / M_{2}^{2}}}{f_{B_{s}} m_{B_{s}} f_{K_{2}^{*}} m_{K_{2}^{*}}^{2}}\left\{\frac{-1}{(2 \pi)^{2}}\right. \\
& \quad \cdot \int_{m_{s}^{2}}^{s_{0}^{\prime}} \int_{s_{L}}^{s_{0}} \rho_{n}\left(s, s^{\prime}, q^{2}\right) e^{-s / M_{1}^{2}} e^{-s^{\prime} / M_{2}^{2}} \\
& \left.\quad+\widetilde{B}\left[C_{n}^{(3)}+C_{n}^{(5)}\right] d s^{\prime} d s\right\},
\end{aligned}
$$

where $n=0, \ldots, 2$ and $s_{0}$ and $s_{0}^{\prime}$ are the continuum thresholds in the initial and final channels, respectively. The lower limit in the integration over $s$ is $s_{L}=m_{b}^{2}+\left(m_{b}^{2} /\left(m_{b}^{2}-q^{2}\right)\right) s^{\prime}$. Also, $\widetilde{B}$ transformation is defined as follows:

$$
\begin{aligned}
\widetilde{B}\left[\frac{1}{\left(p^{2}-m_{b}^{2}\right)^{m}\left(p^{\prime 2}-m_{u}^{2}\right)^{n}}\right] \\
=\frac{(-1)^{m+n}}{\Gamma(n) \Gamma(m)} \frac{e^{-m_{b}^{2} / M_{1}^{2}} e^{-m_{u}^{2} / M_{2}^{2}}}{\left(M_{1}^{2}\right)^{m-1}\left(M_{2}^{2}\right)^{n-1}},
\end{aligned}
$$

where $M_{1}^{2}$ and $M_{2}^{2}$ are Borel mass parameters.

In (20), to subtract the contributions of the higher states and the continuum, the quark-hadron duality assumption is also used; that is, it is assumed that

$$
\rho^{\text {higherstates }}\left(s, s^{\prime}\right)=\rho\left(s, s^{\prime}\right) \theta\left(s-s_{0}\right) \theta\left(s^{\prime}-s_{0}^{\prime}\right) .
$$

We would like to provide the same results for $B \rightarrow a_{2} \ell v$ and $B \rightarrow f_{2} \ell \nu$ decays. With a little bit of change in the above expressions such as $s \leftrightarrow d(u)$ and $m_{K_{2}^{*}} \leftrightarrow m_{a_{2}}\left(m_{f_{2}}\right)$, we can easily find similar results in (20) for the form factors of the new transitions.

\section{Numerical Analysis}

In this section, we numerically analyze the sum rules for the form factors $V\left(q^{2}\right), A_{0}\left(q^{2}\right), A_{1}\left(q^{2}\right)$, and $A_{2}\left(q^{2}\right)$ as well as branching ratio values of the transitions $B\left(B_{s}\right) \rightarrow T$, where $T$ can be one of the tensor mesons $K_{2}^{*}, a_{2}$, or $f_{2}$. The values of the meson masses and leptonic decay constants are chosen as presented in Table 1 . Also, $m_{b}=4.820 \mathrm{GeV}, m_{s}=0.150 \mathrm{GeV}$ [38], $m_{\tau}=1.776 \mathrm{GeV}$, and $m_{\mu}=0.105 \mathrm{GeV}$ [30].

From the 3PSR, it is clear that the form factors also contain the continuum thresholds $s_{0}$ and $s_{0}^{\prime}$ and the Borel parameters $M_{1}^{2}$ and $M_{2}^{2}$ as the main input. These are not physical quantities; hence the form factors should be independent of these parameters. The continuum thresholds, $s_{0}$ and $s_{0}^{\prime}$, are not completely arbitrary, but these are in correlation with the energy of the first exiting state with the same quantum numbers as the considered interpolating currents. The value of the continuum threshold $s_{0}^{B\left(B_{s}\right)}=$ $35 \mathrm{GeV}^{2}$ [39] is calculated from the 3PSR. The values of the continuum threshold $s_{0}^{\prime}$ for the tensor mesons $K_{2}^{*}, a_{2}$, and $f_{2}$ are taken to be $s_{0}^{K_{2}^{*}}=3.13 \mathrm{GeV}^{2}, s_{0}^{a_{2}}=2.70 \mathrm{GeV}^{2}$, and $s_{0}^{f_{2}}=2.53 \mathrm{GeV}^{2}$, respectively [9]. In this work, the variations of $s_{0}^{T}\left(T=a_{2}, K_{2}^{*}, f_{2}\right)$ are considered to be \pm 0.2 . In these regions, the dependence of the form factors on the continuum threshold values is very small. For instance, we have shown the variations of the form factor $A_{1}^{B_{s} \rightarrow K_{2}^{*}}\left(q^{2}\right)$ for different values of $s_{0}^{K_{2}^{*}}$ in Figure 3. As can be seen, these plots are very close to each other.

We search for the intervals of the Borel parameters so that our results are almost insensitive to their variations. One more condition for the intervals of these parameters is the fact that the aforementioned intervals must suppress the higher states, continuum, and contributions of the highest-order operators. In other words, the sum rules for the form factors must converge. As a result, we get $8 \mathrm{GeV}^{2} \leq M_{1}^{2} \leq 12 \mathrm{GeV}^{2}$ and $4 \mathrm{GeV}^{2} \leq M_{2}^{2} \leq 8 \mathrm{GeV}^{2}$. To show how the form factors depend on the Borel mass parameters, as examples, we depict the variations of the form factors $V, A_{0}, A_{1}$, and $A_{2}$ for $B_{s} \rightarrow K_{2}^{*} \ell v$ at $q^{2}=0$ with respect to the variations of the $M_{1}^{2}$ and $M_{2}^{2}$ parameters in their working regions in Figure 4. From these figures, it is revealed that the form factors weakly depend on these parameters in their working regions.

In the Borel transform scheme, the ratio of the nonperturbative to perturbative part of the form factor $V^{B_{s} \rightarrow K_{2}^{*}}$ is about $V^{\text {non-per }}(0) / V^{\text {per }}(0) \simeq 13 \%$. This value confirms that the higher order corrections are small, constituting a few percent, and can easily be neglected. Our calculation shows that the same suppression is observed for all other form factors.

The sum rules for the form factors are truncated at about $0 \leq q^{2} \leq 11 \mathrm{GeV}^{2}$. The dependence of the form factors $V, A_{0}$, 


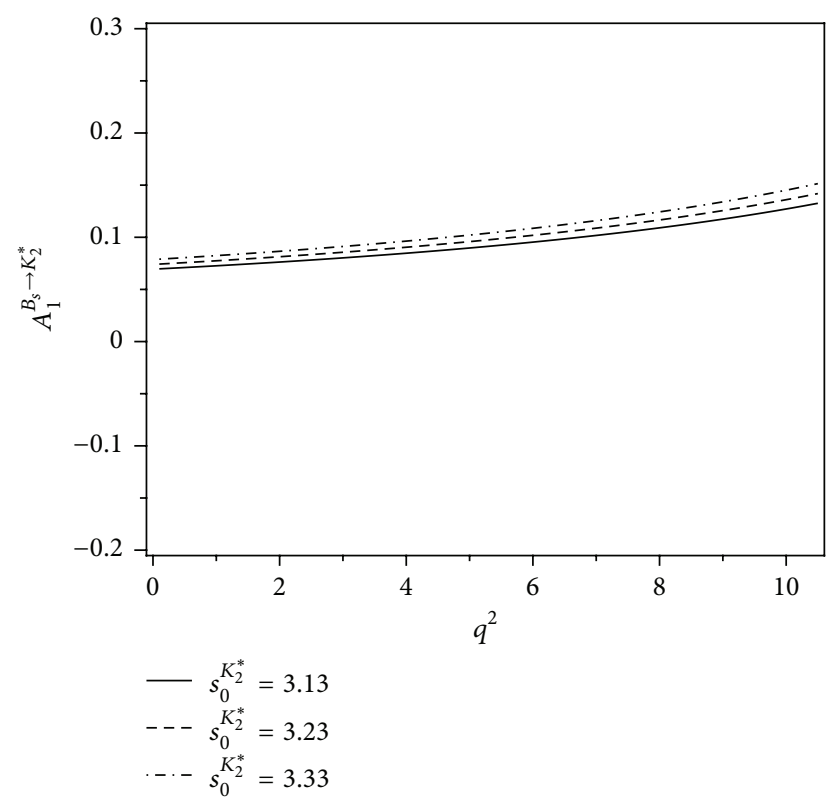

FIgURE 3: The form factor of $A_{1}^{B_{s} \rightarrow K_{2}^{*}}$ on $q^{2}$ for different values of $s_{0}^{K_{2}^{*}}$.

$A_{1}$, and $A_{2}$ on $q^{2}$ for $B \rightarrow T$ transitions is shown in Figure 5 . However, it is necessary to obtain the behavior of the form factors with respect to $q^{2}$ in the full physical region, $0 \leq q^{2} \leq$ $\left(m_{B\left(B_{s}\right)}-m_{T}\right)^{2}$, in order to calculate the decay width of the $B \rightarrow T$ transitions. So, to extend our results, we look for a parametrization of the form factors in such a way that in the region $0 \leq q^{2} \leq\left(m_{B\left(B_{s}\right)}-m_{T}\right)^{2}$, this parametrization coincides with the sum rules predictions. Our numerical calculations show that the sufficient parametrization of the form factors with respect to $q^{2}$ is as follows:

$$
f\left(q^{2}\right)=\frac{f(0)}{1-a\left(q^{2} / m_{B\left(B_{s}\right)}^{2}\right)+b\left(q^{2} / m_{B\left(B_{s}\right)}^{2}\right)^{2}} .
$$

The values of the parameters $f(0), a$, and $b$ for the transition form factors of $B \rightarrow T$ are given in Table 2 .

In Table 3, our results for the form factors of $B \rightarrow T \ell v$ decays in $q^{2}=0$ are compared with those of other approaches such as the LCSR, the PQCD, the LEET, and the ISGW II model. Our results are in good agreement with those of the LCSR, PQCD, and LEET in all cases.

At the end of this section, we would like to present the differential decay widths of the process under consideration. Using the parametrization of these transitions in terms of the form factors, the differential decay width for $B \rightarrow T \ell v$ transition is obtained as

$$
\begin{aligned}
& \frac{d \Gamma(B \rightarrow T \ell \nu)}{d q^{2}} \\
& =\frac{\left|G_{F} V_{u b}\right|^{2} \sqrt{\lambda\left(m_{B}^{2}, m_{T}^{2}, q^{2}\right)}}{256 m_{B}^{3} \pi^{3} q^{2}}\left(1-\frac{m_{\ell}^{2}}{q^{2}}\right)^{2} \\
& \cdot\left(X_{L}+X_{+}+X_{-}\right),
\end{aligned}
$$

TABLE 2: Parameter values appearing in the fit functions of the $B \rightarrow$ Tev decays.

\begin{tabular}{lccc}
\hline Form factor & $f(0)$ & $a$ & $b$ \\
\hline$V^{B_{s} \rightarrow K_{2}^{*}}$ & 0.13 & 2.19 & 0.83 \\
$A_{1}^{B_{S} \rightarrow K_{2}^{*}}$ & 0.10 & 1.36 & 0.09 \\
$V^{B \rightarrow a_{2}}$ & 0.13 & 2.10 & 0.75 \\
$A_{1}^{B \rightarrow a_{2}}$ & 0.11 & 1.45 & 0.23 \\
$V^{B \rightarrow f_{2}}$ & 0.12 & 2.01 & 0.60 \\
$A_{1}^{B \rightarrow f_{2}}$ & 0.10 & 1.40 & 0.16 \\
$A_{0}^{B_{S} \rightarrow K_{2}^{*}}$ & 0.23 & 3.77 & 4.21 \\
$A_{2}^{B_{s} \rightarrow K_{2}^{*}}$ & 0.05 & 0.21 & -2.99 \\
$A_{0}^{B \rightarrow a_{2}}$ & 0.26 & 3.71 & 4.03 \\
$A_{2}^{B \rightarrow a_{2}}$ & 0.09 & 0.63 & 0.46 \\
$A_{0}^{B \rightarrow f_{2}}$ & 0.24 & 3.70 & 4.02 \\
$A_{2}^{B \rightarrow f_{2}}$ & 0.09 & 0.46 & 0.29 \\
\hline
\end{tabular}

where $m_{\ell}$ represents the mess of the charged lepton. The other parameters are defined as

$$
\begin{aligned}
& X_{L}=\frac{1}{9} \frac{\lambda}{m_{T}^{2} m_{B}^{2}}\left[\left(2 q^{2}+m_{\ell}^{2}\right) h_{0}^{2}\left(q^{2}\right)+3 \lambda m_{\ell}^{2} A_{0}^{2}\left(q^{2}\right)\right], \\
& X_{ \pm}=\frac{2 q^{2}}{3}\left(2 q^{2}+m_{\ell}^{2}\right) \frac{\lambda}{8 m_{T}^{2} m_{B}^{2}}\left[\left(m_{B}+m_{T}\right) A_{1}\left(q^{2}\right)\right. \\
&\left.\mp \frac{\sqrt{\lambda}}{m_{B}+m_{T}} V\left(q^{2}\right)\right]^{2}, \\
& h_{0}\left(q^{2}\right)=\frac{1}{2 m_{T}}\left[\left(m_{B}^{2}-m_{T}^{2}-q^{2}\right)\left(m_{B}+m_{T}\right) A_{1}\left(q^{2}\right)\right. \\
&\left.-\frac{\lambda}{m_{B}+m_{T}} A_{2}\left(q^{2}\right)\right] .
\end{aligned}
$$

Integrating (24) over $q^{2}$ in the whole physical region and using $V_{u b}=(3.89 \pm 0.44) \times 10^{-3}$ [30], the branching ratios of the $B \rightarrow T \ell \nu$ are obtained. The differential branching ratios of the $B \rightarrow T \ell v$ decays on $q^{2}$ are shown in Figure 6. The branching ratio values of these decays are also obtained as presented in Table 4. Furthermore, this table contains the results estimated via the PQCD. Considering the uncertainties, our estimations for the branching ratio values of the $B \rightarrow T \ell \nu$ decays are in consistent agreement with those of the PQCD.

It should be noted that the uncertainties in the branching ratio values come from the form factors, the CKM parameter, and the meson and lepton masses which are about $30 \%$ of the central values.

In summary, we considered $B_{s}(B) \rightarrow K_{2}^{*}\left(a_{2}, f_{2}\right) \ell v$ channels and computed the relevant form factors considering the contribution of the quark condensate corrections. Our results are in good agreement with those of the LCSR, PQCD, and LEET in all cases. We also evaluated the total decays widths and the branching ratios of these decays. Our branching ratio values of these decays are in consistent agreement with those of the PQCD. 
TABLE 3: Comparison of the form factor values of $B \rightarrow T \ell v$ decays in $q^{2}=0$ in different approaches.

\begin{tabular}{|c|c|c|c|c|c|}
\hline Form factor & This work & LCSR [25] & PQCD [5] & LEET [26-28] & ISGW II [29] \\
\hline$V^{B_{s} \rightarrow K_{2}^{*}}$ & $0.13 \pm 0.03$ & $0.15 \pm 0.02$ & $0.18_{-0.04}^{+0.05}$ & - & - \\
\hline$A_{0}^{B_{s} \rightarrow K_{2}^{*}}$ & $0.23 \pm 0.06$ & $0.22 \pm 0.04$ & $0.15_{-0.03}^{+0.04}$ & - & - \\
\hline$A_{1}^{B_{s} \rightarrow K_{2}^{*}}$ & $0.10 \pm 0.02$ & $0.12 \pm 0.02$ & $0.11_{-0.02}^{+0.03}$ & - & - \\
\hline$A_{2}^{B_{s} \rightarrow K_{2}^{*}}$ & $0.05 \pm 0.01$ & $0.05 \pm 0.02$ & $0.07_{-0.02}^{+0.02}$ & - & - \\
\hline$V^{B \rightarrow a_{2}}$ & $0.13 \pm 0.03$ & $0.18 \pm 0.02$ & $0.18_{-0.04}^{+0.05}$ & $0.18 \pm 0.03$ & 0.32 \\
\hline$A_{0}^{B \rightarrow a_{2}}$ & $0.26 \pm 0.07$ & $0.21 \pm 0.04$ & $0.18_{-0.04}^{+0.06}$ & $0.14 \pm 0.02$ & 0.20 \\
\hline$A_{1}^{B \rightarrow a_{2}}$ & $0.11 \pm 0.04$ & $0.14 \pm 0.02$ & $0.11_{-0.03}^{+0.03}$ & $0.13 \pm 0.02$ & 0.16 \\
\hline$A_{2}^{B \rightarrow a_{2}}$ & $0.09 \pm 0.02$ & $0.09 \pm 0.02$ & $0.06_{-0.01}^{+0.02}$ & $0.13 \pm 0.02$ & 0.14 \\
\hline$V^{B \rightarrow f_{2}}$ & $0.12 \pm 0.04$ & $0.18 \pm 0.02$ & $0.12_{-0.03}^{+0.03}$ & $0.18 \pm 0.02$ & 0.32 \\
\hline$A_{0}^{B \rightarrow f_{2}}$ & $0.24 \pm 0.06$ & $0.20 \pm 0.04$ & $0.13_{-0.03}^{+0.04}$ & $0.13 \pm 0.02$ & 0.20 \\
\hline$A_{1}^{B \rightarrow f_{2}}$ & $0.10 \pm 0.02$ & $0.14 \pm 0.02$ & $0.08_{-0.02}^{+0.02}$ & $0.12 \pm 0.02$ & 0.16 \\
\hline$A_{2}^{B \rightarrow f_{2}}$ & $0.09 \pm 0.02$ & $0.10 \pm 0.02$ & $0.04_{-0.01}^{+0.01}$ & $0.13 \pm 0.02$ & 0.14 \\
\hline
\end{tabular}
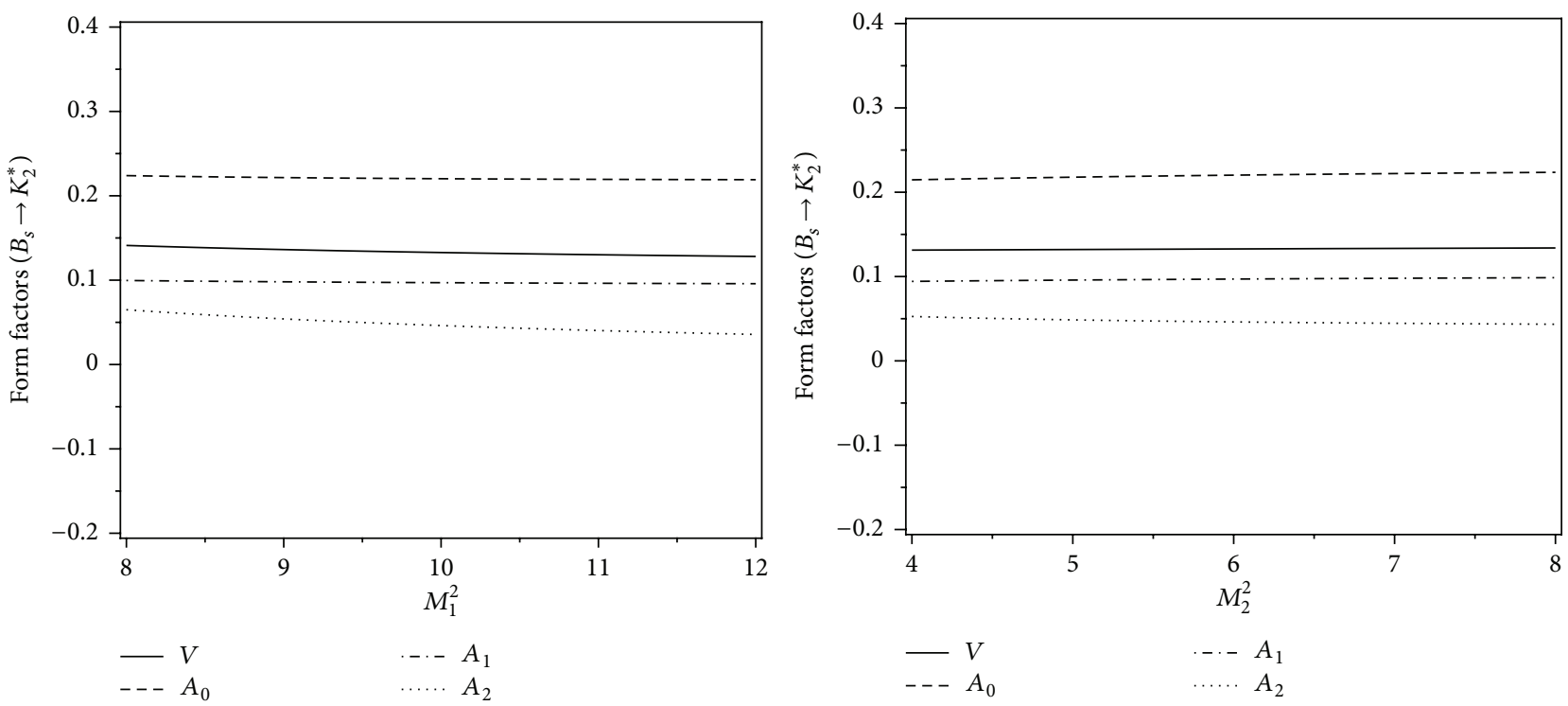

FIgURE 4: The form factor of $B_{s} \rightarrow K_{2}^{*}$ on $M_{1}^{2}$ and $M_{2}^{2}$.

TABLE 4: Comparison of the branching ratio values of $B \rightarrow T \ell v$ decays with those of the PQCD (in units of $10^{-4}$ ).

\begin{tabular}{lcc}
\hline & This work & PQCD [5] \\
\hline $\operatorname{Br}\left(B \rightarrow a_{2} \mu \nu\right)$ & $0.82 \pm 0.25$ & $1.16_{-0.57}^{+0.81}$ \\
$\operatorname{Br}\left(B_{s} \rightarrow K_{2}^{*} \mu \nu\right)$ & $0.65 \pm 0.20$ & $0.73_{-0.33}^{+0.48}$ \\
$\operatorname{Br}\left(B \rightarrow f_{2} \mu \nu\right)$ & $0.77 \pm 0.23$ & $0.69_{-0.34}^{+0.48}$ \\
$\operatorname{Br}\left(B \rightarrow a_{2} \tau \nu\right)$ & $0.51 \pm 0.17$ & $0.41_{-0.20}^{+0.29}$ \\
$\operatorname{Br}\left(B_{s} \rightarrow K_{2}^{*} \tau \nu\right)$ & $0.35 \pm 0.11$ & $0.25_{-0.12}^{+0.17}$ \\
$\operatorname{Br}\left(B \rightarrow f_{2} \tau \nu\right)$ & $0.53 \pm 0.18$ & $0.25_{-0.13}^{+0.18}$ \\
\hline
\end{tabular}

\section{Appendix}

In this appendix, the explicit expressions of the coefficients $\lambda\left(s, s^{\prime}, q^{2}\right), B_{l}(l=1,2), D_{j}(j=1, \ldots, 4)$, and $E_{r}(r=$ $1, \ldots, 6)$ are given.

$$
\lambda\left(s, s^{\prime}, q^{2}\right)=s^{2}+s^{\prime 2}+\left(q^{2}\right)^{2}-2 s q^{2}-2 s^{\prime} q^{2}-2 s s^{\prime},
$$

$B_{1}=\frac{I_{0}}{\lambda\left(s, s^{\prime}, q^{2}\right)}\left[2 s^{\prime} \Delta-\Delta^{\prime} u\right]$,

$B_{2}=\frac{I_{0}}{\lambda\left(s, s^{\prime}, q^{2}\right)}\left[2 s \Delta^{\prime}-\Delta u\right]$

$D_{1}=-\frac{I_{0}}{2 \lambda\left(s, s^{\prime}, q^{2}\right)}\left[4 s s^{\prime} m_{s}^{2}-s \Delta^{\prime 2}-s^{\prime} \Delta^{2}-u^{2} m_{s}^{2}\right.$

$\left.+u \Delta \Delta^{\prime}\right]$

$D_{2}=-\frac{I_{0}}{\lambda^{2}\left(s, s^{\prime}, q^{2}\right)}\left[8 s s^{\prime 2} m_{s}^{2}-2 s s^{\prime} \Delta^{\prime}-6 s^{\prime 2} \Delta^{2}\right.$

$\left.-2 u^{2} s^{\prime} m_{s}^{2}+6 s^{\prime} u \Delta \Delta^{\prime}-u^{2} \Delta^{\prime 2}\right]$,

$D_{3}=\frac{I_{0}}{\lambda^{2}\left(s, s^{\prime}, q^{2}\right)}\left[4 s s^{\prime} u m_{s}^{2}+4 s s^{\prime} \Delta \Delta^{\prime}-3 s u \Delta^{\prime 2}\right.$

$\left.-3 u \Delta^{2} s^{\prime}-u^{3} m_{3}^{2}+2 u^{2} \Delta \Delta^{\prime}\right]$, 

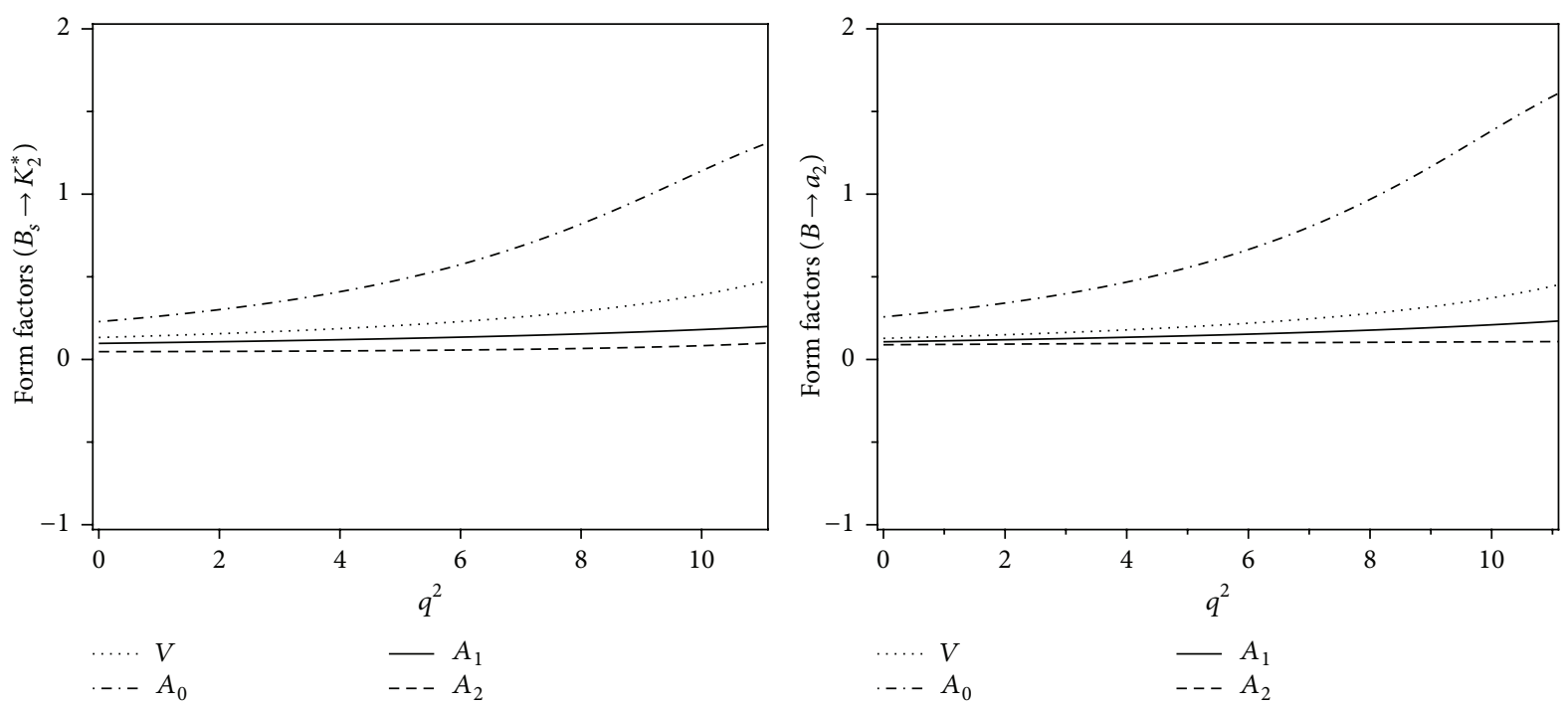

$-A_{1}$

$\begin{array}{ll}\cdots & V \\ \cdots & A_{0}\end{array}$

$-A_{1}$
$---A_{2}$



FIGURE 5: The SR predictions for the form factors of the $B\left(B_{s}\right) \rightarrow T \ell \nu$ transitions on $q^{2}$.
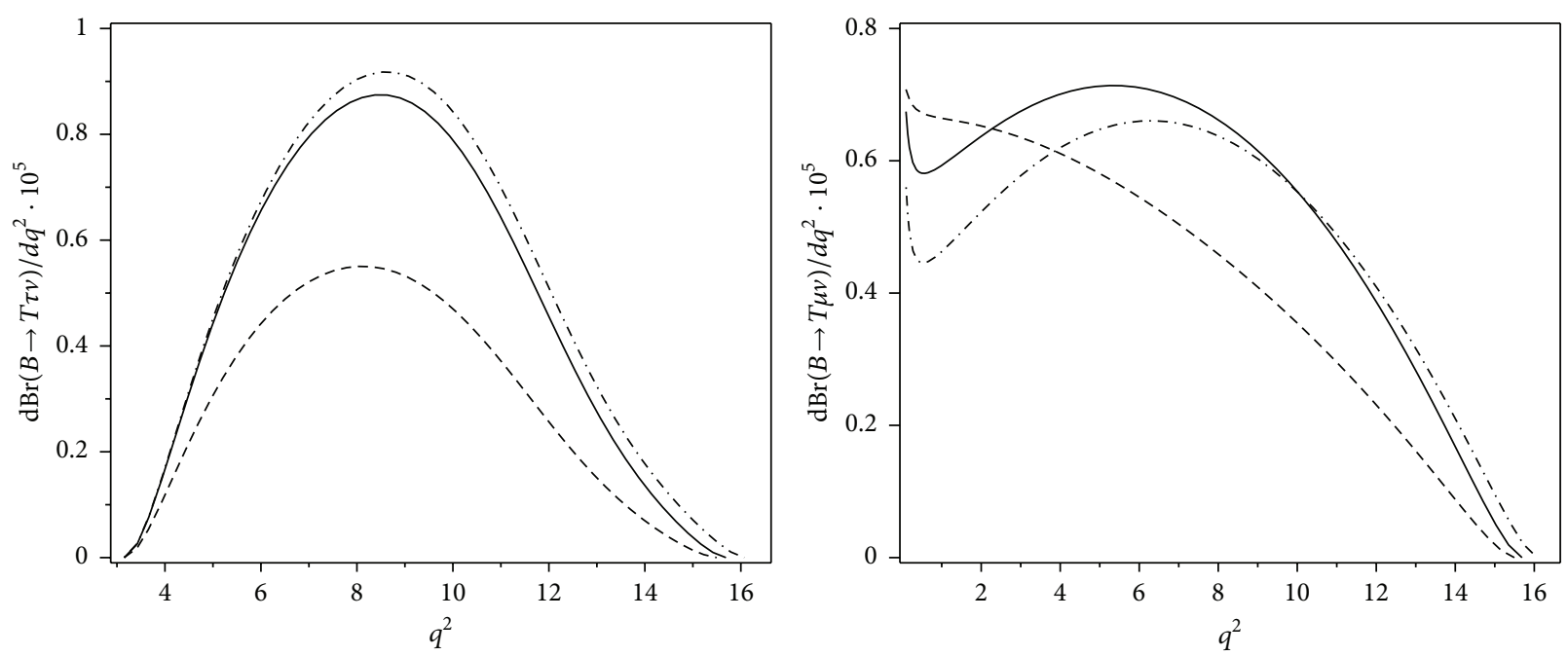

$\begin{array}{ll}- & a_{2} \\ --- & K_{2}^{*} \\ -- & f_{2}\end{array}$

$\begin{array}{ll}- & a_{2} \\ --- & K_{2}^{*} \\ \ldots & f_{2}\end{array}$

FIgURE 6: The differential branching ratios of the semileptonic $B \rightarrow T \ell \nu$ decays on $q^{2}$. 


$$
\begin{aligned}
& D_{4}=\frac{I_{0}}{\lambda^{2}\left(s, s^{\prime}, q^{2}\right)}\left[-6 s^{\prime} u \Delta \Delta^{\prime}+6 s^{2} \Delta^{\prime 2}-8 s^{2} s^{\prime} m_{s}^{2}\right. \\
& \left.+2 u^{2} s m_{s}^{2}+u^{2} \Delta^{2}+2 s s^{\prime} \Delta^{2}\right] \\
& E_{1}=\frac{I_{0}}{2 \lambda^{2}\left(s, s^{\prime}, q^{2}\right)}\left[8 s^{\prime 2} m_{s}^{2} \Delta s-2 s^{\prime} m_{s}^{2} \Delta u^{2}\right. \\
& -4 u m_{s}^{2} \Delta^{\prime} s s^{\prime}+u^{3} m_{s}^{2} \Delta^{\prime}-2 s^{\prime 2} \Delta^{3}+3 s^{\prime} u \Delta^{2} \Delta^{\prime} \\
& \left.-2 \Delta^{\prime 2} \Delta s s^{\prime}-\Delta^{\prime 2} \Delta u^{2}+u s \Delta^{3}\right] \\
& E_{2}=\frac{I_{0}}{2 \lambda^{2}\left(s, s^{\prime}, q^{2}\right)}\left[8 s^{2} m_{s}^{2} \Delta^{\prime} s^{\prime}-2 s^{2} \Delta^{\prime 3}-4 u m_{s}^{2} \Delta s s^{\prime}\right. \\
& -2 \Delta^{2} \Delta^{\prime} s s^{\prime}+3 u s \Delta^{\prime 2} \Delta-2 s m_{s}^{2} \Delta^{\prime} u^{2}+s^{\prime} u \Delta^{3} \\
& \left.+u^{3} m_{s}^{2} \Delta-\Delta^{2} \Delta^{\prime} u^{2}\right] \\
& E_{3}=-\frac{I_{0}}{\lambda^{3}\left(s, s^{\prime}, q^{2}\right)}\left[48 s m_{s}^{2} \Delta s^{\prime 3}-24 s s^{\prime 2} u m_{s}^{2} \Delta^{\prime}\right. \\
& -12 s s^{\prime 2} \Delta^{\prime 2} \Delta+6 s u \Delta^{\prime 3} s^{\prime}-20 s^{\prime 3} \Delta^{3}+30 s^{\prime 2} u \Delta^{2} \Delta^{\prime} \\
& -12 s^{\prime 2} m_{s}^{2} \Delta u^{2}-12 s^{\prime} \Delta^{\prime 2} \Delta u^{2}+6 s^{\prime} u^{3} m_{s}^{2} \Delta^{\prime} \\
& \left.+u^{3} \Delta^{13}\right] \\
& E_{4}=-\frac{I_{0}}{\lambda^{3}\left(s, s^{\prime}, q^{2}\right)}\left[16 s^{2} m_{s}^{2} \Delta^{\prime} s^{\prime 2}-4 s^{2} \Delta^{\prime 3} s^{\prime}\right. \\
& -12 s s^{\prime 2} \Delta^{2} \Delta^{\prime}-24 s s^{\prime 2} u m_{s}^{2} \Delta+3 u^{3} \Delta^{\prime 2} \Delta \\
& +18 s u \Delta^{\prime 2} \Delta s^{\prime}-4 s \Delta^{\prime 3} u^{2}+10 s^{\prime 2} u \Delta^{3}+6 s^{\prime} u^{3} m_{s}^{2} \Delta \\
& \left.-12 s^{\prime} \Delta^{2} \Delta^{\prime} u^{2}-2 m_{s}^{2} \Delta^{\prime} u^{4}+4 s s^{\prime} u^{2} m_{s}^{2} \Delta^{\prime}\right], \\
& E_{5}=-\frac{I_{0}}{\lambda^{3}\left(s, s^{\prime}, q^{2}\right)}\left[16 s^{2} m_{s}^{2} \Delta s^{\prime 2}-24 s^{2} s^{\prime} u m_{s}^{2} \Delta^{\prime}\right. \\
& -12 s^{2} s^{\prime} \Delta^{\prime 2} \Delta+10 u s^{2} \Delta^{\prime 3}-4 s s^{\prime 2} \Delta^{3}+4 s s^{\prime} u^{2} m_{s}^{2} \Delta \\
& +18 s u \Delta^{2} \Delta^{\prime} s^{\prime}+6 s u^{3} m_{s}^{2} \Delta^{\prime}-12 s \Delta^{2} \Delta u^{2}-4 s^{\prime} \Delta^{3} u^{2} \\
& \left.-2 m_{s}^{2} \Delta u^{4}+3 u^{3} \Delta^{2} \Delta^{\prime}\right] \\
& E_{6}=-\frac{I_{0}}{\lambda^{3}\left(s, s^{\prime}, q^{2}\right)}\left[48 s^{3} m_{s}^{2} \Delta^{\prime} s^{\prime}-20 s^{3} \Delta^{\prime 3}\right. \\
& -12 s^{2} \Delta^{2} \Delta^{\prime} s^{\prime}-24 s^{2} s^{\prime} u m_{s}^{2} \Delta-12 s^{2} m_{s}^{2} \Delta^{\prime} u^{2} \\
& +30 u s^{2} \Delta^{\prime 2} \Delta+6 s u \Delta^{3} s^{\prime}-12 s \Delta^{2} \Delta^{\prime} u^{2}+6 s u^{3} m_{s}^{2} \Delta \\
& \left.+u^{3} \Delta^{3}\right] \\
& \Delta=s+m_{s}^{2}-m_{b}^{2}, \Delta^{\prime}=s^{\prime}+m_{s}^{2}-m_{u}^{2}, u=s+s^{\prime}-q^{2} .
\end{aligned}
$$

\section{Competing Interests}

The authors declare that they have no competing interests.

\section{Acknowledgments}

Partial support of the Isfahan University of Technology Research Council is appreciated.

\section{References}

[1] B. Aubert, Y. Karyotakis, J. P. Lees et al., "Observation of $B$ meson decays to $\omega K^{*}$ and improved measurements for $\omega \rho$ and $\omega f_{0}$," Physical Review D, vol. 79, no. 5, Article ID 052005, 9 pages, 2009.

[2] B. Aubert, M. Bona, Y. Karyotakis et al., "Observation and polarization measurements of $B^{ \pm} \rightarrow \phi K_{1}^{ \pm}$and $B^{ \pm} \rightarrow \phi K_{2}^{* \pm}$," Physical Review Letters, vol. 101, no. 16, Article ID 161801, 2008.

[3] B. Aubert, M. Bona, Y. Karyotakis et al., "Time-dependent and time-integrated angular analysis of $B \rightarrow \varphi K_{S}^{0} \pi^{0}$ and $\varphi K^{ \pm} \pi^{\mp}$," Physical Review D, vol. 78, no. 9, Article ID 092008, 27 pages, 2008.

[4] E. Barberio, R. Bernhard, S. Blyth et al., "Averages of b-hadron and c-hadron properties at the end of 2007," https://arxiv.org/abs/0808.1297.

[5] W. Wang, " $B$ to tensor meson form factors in the perturbative QCD approach," Physical Review D, vol. 83, no. 1, Article ID 014008, 13 pages, 2011.

[6] S. V. Dombrowski, "Experimental status of scalar and tensor mesons," Nuclear Physics B-Proceedings Supplements, vol. 56, no. 1-2, pp. 125-135, 1997.

[7] C. Amsler, M. Doser, M. Antonelli et al., "Review of particle physics," Physics Letters B, vol. 667, no. 1-5, pp. 1-6, 2008.

[8] D. M. Li, H. Yu, and Q. X. Shen, "Properties of the tensor mesons $f_{2}(1270)$ and $f_{2}^{\prime}(1525)$," Journal of Physics G: Nuclear and Particle Physics, vol. 27, no. 4, pp. 807-813, 2001.

[9] H.-Y. Cheng, Y. Koike, and K.-C. Yang, "Two-parton light-cone distribution amplitudes of tensor mesons," Physical Review D, vol. 82, no. 5, Article ID 054019, 7 pages, 2010.

[10] K. Azizi, H. Sundu, and S. Şahin, "Semileptonic $B_{s} \rightarrow$ $D_{s 2}^{*}(2573) \ell \bar{v}_{\ell}$ transition in QCD," The European Physical Journal C, vol. 75, article 197, pp. 1-13, 2015.

[11] P. Ball, "Semileptonic decays $D \rightarrow \pi(\rho) e v$ and $B \rightarrow \pi(\rho) e v$ from QCD sum rules," Physical Review D, vol. 48, no. 7, pp. 3190-3203, 1993.

[12] P. Colangelo, F. De Fazio, P. Santorelli, and E. Scrimieri, "QCD sum rule analysis of the decays $B \rightarrow K \ell^{+} \ell^{-}$and $B \rightarrow K^{*} \ell^{+} \ell^{-}$," Physical Review D, vol. 53, no. 7, pp. 3672-3686, 1996.

[13] P. Colangelo, F. De Fazio, P. Santorelli, and E. Scrimieri, "Rare $B \rightarrow K\left(^{*}\right) \nu v$ decays at B factories," Physics Letters B, vol. 395, no. 3-4, pp. 339-344, 1997.

[14] P. Colangelo, C. A. Dominguez, G. Nardulli, and N. Paver, "Radiative $B \rightarrow K * \gamma$ transition in QCD," Physics Letters B, vol. 317, no. 1-2, pp. 183-189, 1993.

[15] M.-Z. Yang, "Semileptonic decay of $B$ and $D \rightarrow K_{0}^{*}(1430) \bar{\ell} v$ from QCD sum rule," Physical Review D, vol. 73, no. 3, Article ID 034027, 9 pages, 2006.

[16] N. Gharamany and R. Khosravi, "Analysis of the rare semileptonic decays of $B_{s}$ to $f_{0}(980)$ and $K_{0}^{*}(1430)$ scalar mesons in QCD sum rules," Physical Review D, vol. 80, no. 1, Article ID 016009, 2009.

[17] R. Khosravi, "Form factors and branching ratios of the FCNC $B \rightarrow a_{1} \ell^{+} \ell^{-}$decays," The European Physical Journal C, vol. 75, article 220, 2015. 
[18] P. Ball, “The decay $B \rightarrow K^{*} \gamma$ from QCD sum rules," https:// arxiv.org/abs/hep-ph/9308244.

[19] T. E. Coan, V. Fadeyev, Y. Maravin et al., "Study of exclusive radiative $B$ meson decays," Physical Review Letters, vol. 84, no. 23, pp. 5283-5287, 2000.

[20] S. Ahmed, M. S. Alam, S. B. Athar et al., " $b \rightarrow s \gamma$ branching fraction and CP asymmetry," https://arxiv.org/abs/hep-ex/9908022.

[21] R. Barate, D. Buskulic, D. Decamp et al., "A measurement of the inclusive $b \rightarrow s \gamma$ branching ratio," Physics Letters B, vol. 429, no. 1-2, pp. 169-187, 1998.

[22] A. Abada, C. R. Allton, Ph. Boucaud et al., "Semi-leptonic decays of heavy flavours on a fine grained lattice," Nuclear Physics B, vol. 416, no. 2, pp. 675-695, 1994.

[23] C. R. Allton, M. Crisafulli, V. Lubicz et al., "Lattice calculation of $D$ - and $B$-meson semileptonic decays, using the clover action at $\beta=6.0$ on APE," Physics Letters B, vol. 345, no. 4, pp. 513-523, 1995.

[24] D. R. Burford, H. D. Duong, J. M. Flynn et al., "Form factors for $B \rightarrow \pi l \nu l$ and $B \rightarrow K * \gamma$ decays on the lattice," Nuclear Physics $B$, vol. 447, no. 2-3, pp. 425-437, 1995.

[25] K. C. Yang, " $B$ to light tensor meson form factors derived from light-cone sum rules," Physics Letters B, vol. 695, no. 5, pp. 444$448,2011$.

[26] J. Charles, A. Le. Yaouanc, L. Oliver, O. Pène, and J.-C. Raynal, "Heavy-to-light form factors in the final hadron large energy limit: covariant quark model approach," Physics Letters B, vol. 451, no. 1-2, pp. 187-194, 1999.

[27] D. Ebert, R. N. Faustov, and V. O. Galkin, "Form factors of heavy-to-light B decays at large recoil," Physical Review D, vol. 64, no. 9, Article ID 094022, 2001.

[28] A. Datta, Y. Gao, A. V. Gritsan, D. London, M. Nagashima, and A. Szynkman, "Study of polarization in $B \rightarrow V T$ decays," Physical Review D, vol. 77, no. 11, Article ID 114025, 2008.

[29] D. Scora and N. Isgur, "Semileptonic meson decays in the quark model: an update," Physical Review D, vol. 52, no. 5, pp. 27832812, 1995.

[30] J. Beringer, J.-F. Arguin, R. M. Barnett et al., "Review of particle physics," Physical Review D, vol. 86, no. 1, Article ID 010001, 2012.

[31] E. Gámiz, C. T. H. Davies, G. P. Lepage, J. Shigemitsu, and M. Wingate, "Neutral $B$ meson mixing in unquenched lattice QCD," Physical Review D, vol. 80, no. 1, Article ID 014503, 2009.

[32] M. J. Baker, J. Bordes, C. A. Dominguez, J. Penarrocha, and K. Schilcher, " $B$ meson decay constants $f_{B c}, f_{B s}$ and $f_{B}$ from QCD sum rules," Journal of High Energy Physics, vol. 2014, no. 7, article 32, 2014.

[33] T. M. Aliev and M. A. Shifman, "Old tensor mesons in QCD sum rules," Physics Letters B, vol. 112, no. 4-5, pp. 401-405, 1982.

[34] P. Ball and R. Zwicky, "New results on $B \rightarrow \pi, K, \eta$ decay form factors from light-cone sum rules," Physical Review D, vol. 71, no. 1, Article ID 014015, 2005.

[35] P. Colangelo and A. Khodjamirian, "QCD sum rules, a modern perspective," in At the Frontier of Particle Physics: Handbook of QCD, M. Shifman, Ed., vol. 3, p. 1495, World Scientific, Singapore, 2001.

[36] J. Bordes, C. A. Dominguez, P. Moodley, J. Penarrocha, and K. Schilcher, "Chiral corrections to the SU(2) x SU(2) GellMann-Oakes-Renner relation," Journal of High Energy Physics, vol. 2010, p. 64, 2010.
[37] J. Bordes, C. A. Dominguez, P. Moodley, J. Peñarrocha, and K. Schilcher, "Corrections to the $\mathrm{SU}(3) \times \mathrm{SU}(3)$ Gell-MannOakes-Renner relation and chiral couplings $L_{8}^{r}$ and $H_{2}^{r}$," Journal of High Energy Physics, vol. 2012, artice 102, 2012.

[38] M.-Q. Huang, "Exclusive semileptonic $B_{s}$ decays to excited $D_{s}$ mesons: search of $D_{s J}(2317)$ and $D_{s J}(2460)$," Physical Review D, vol. 69, no. 11, Article ID 114015, 10 pages, 2004.

[39] M. A. Shifman, A. I. Vainshtein, and V. I. Zakharov, "QCD and resonance physics. Theoretical foundations," Nuclear Physics B, vol. 147, no. 5, pp. 385-447, 1979. 

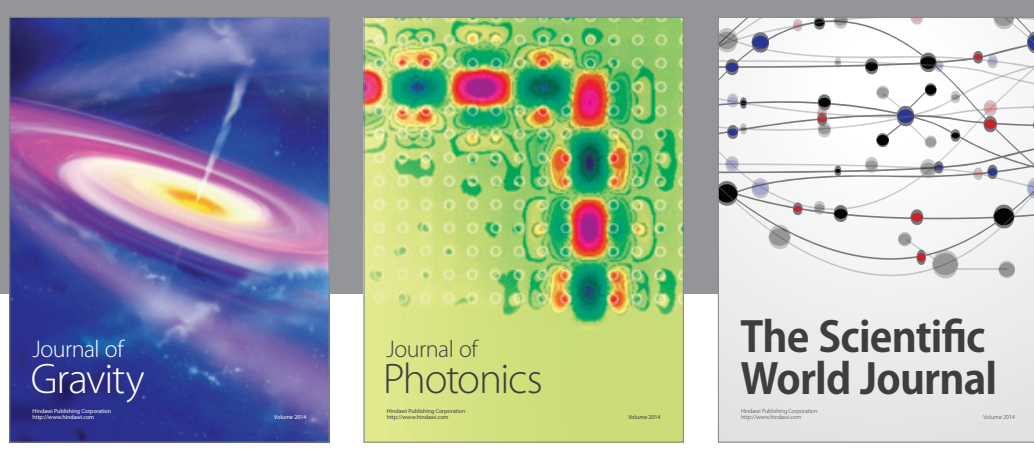

The Scientific World Journal
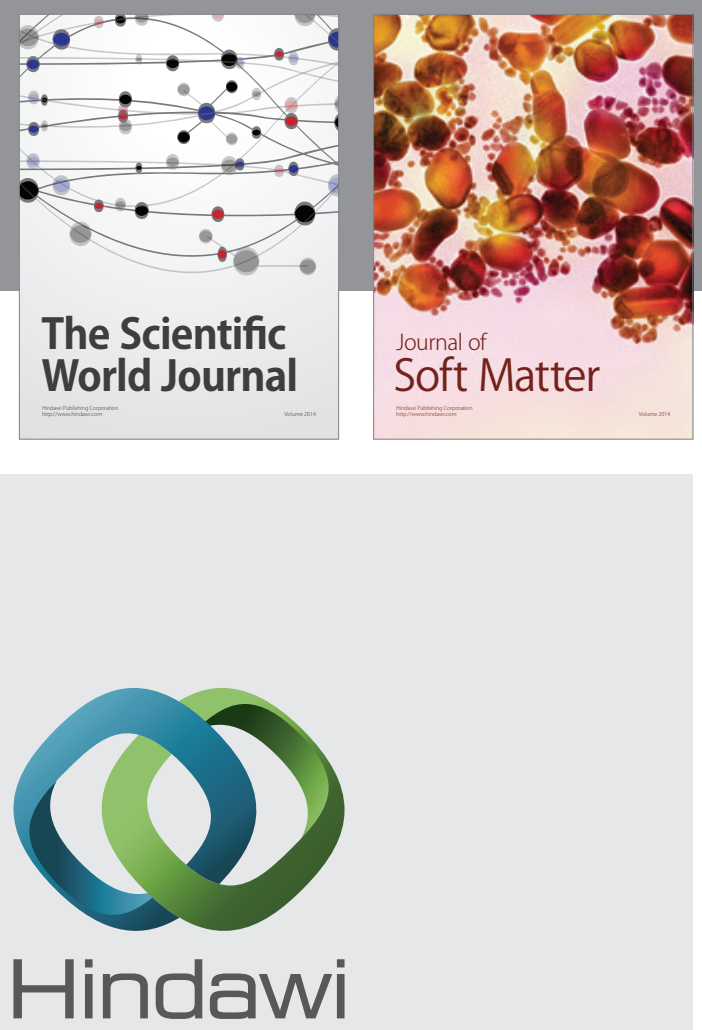

Submit your manuscripts at

http://www.hindawi.com

nternational Journal of

Statistical Mechanics
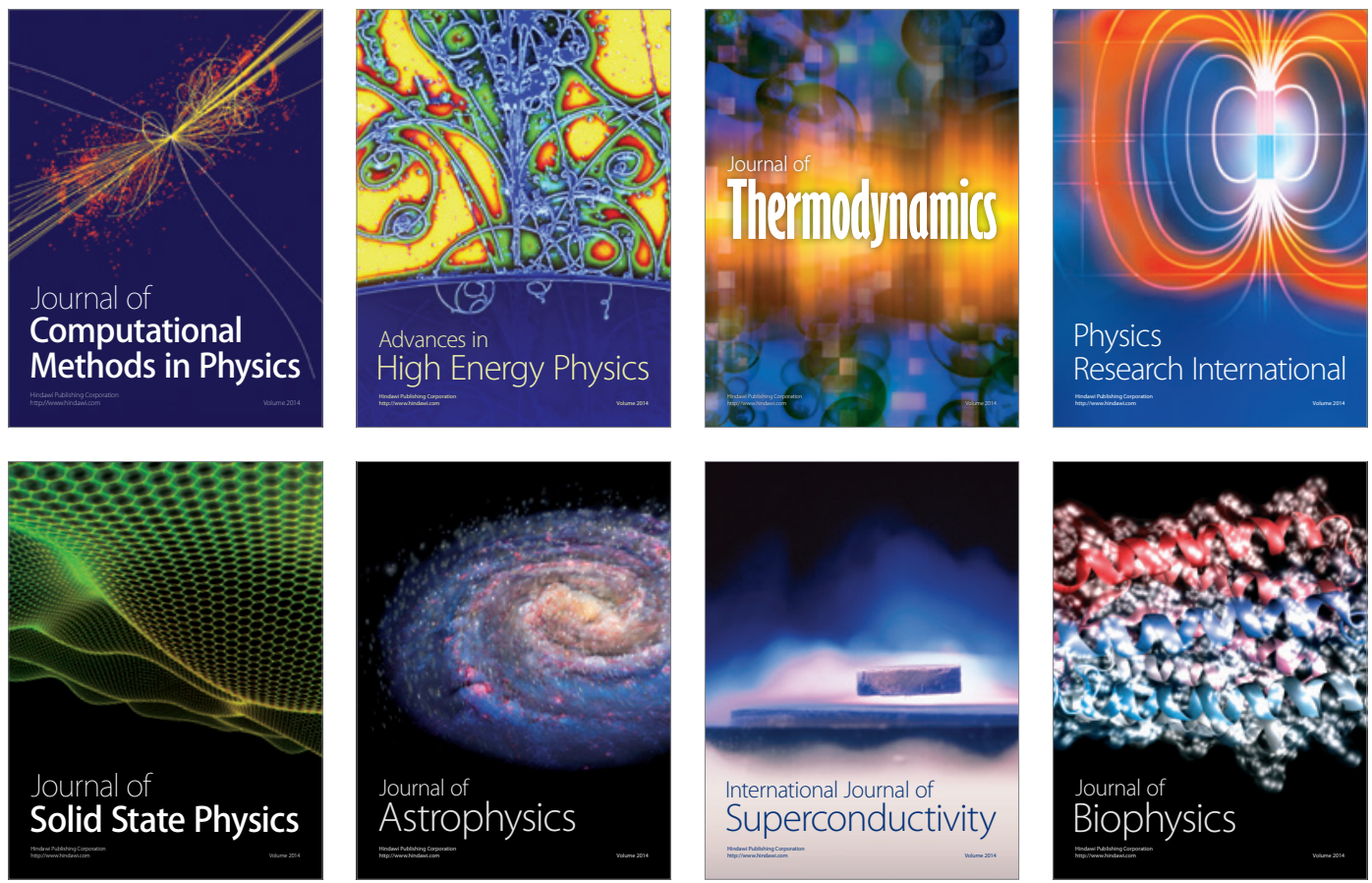
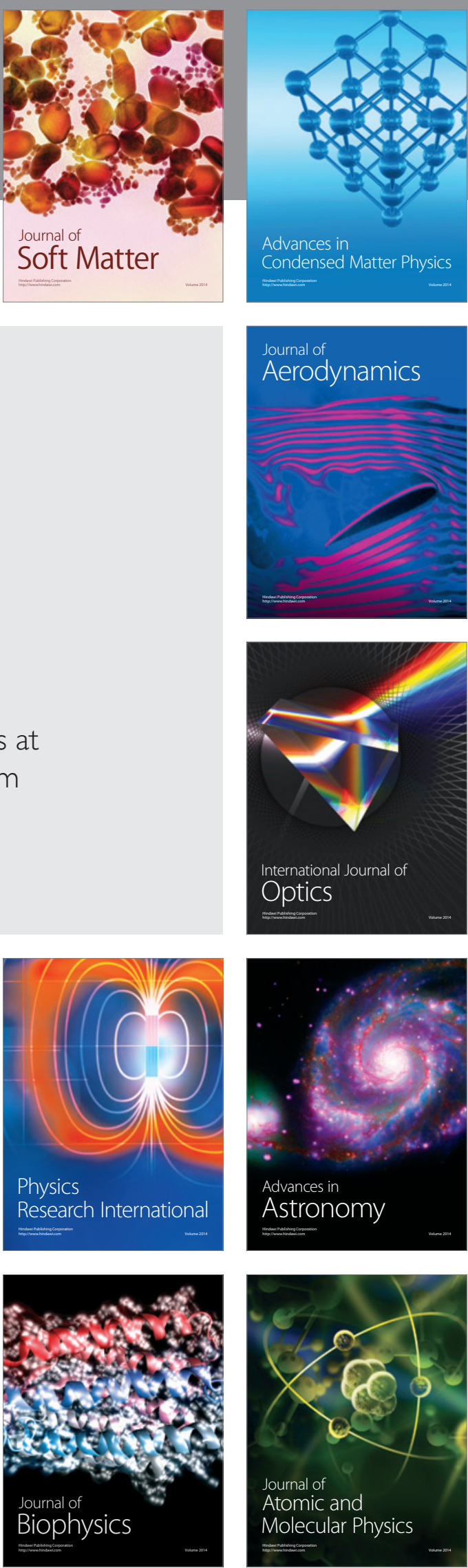